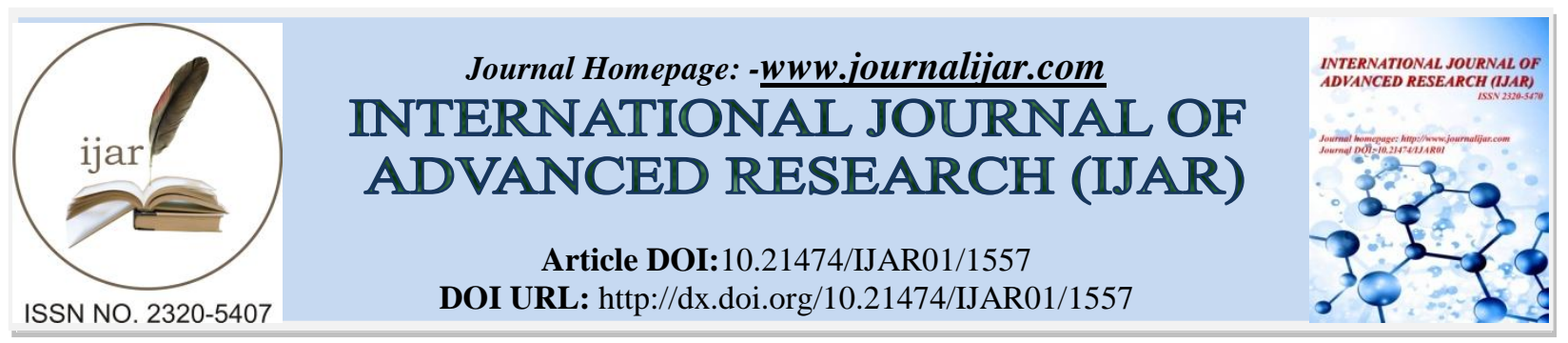

RESEARCH ARTICLE

\title{
COMPARATIVE STUDY OF THE PREVALENCE OF MUSCULOSKELETAL DISORDERS IN DENTAL UNDERGRADUATE AND POSTGRADUATE STUDENTS.
}

Jee Hun Kim ${ }^{1}$, Nakul R Raval ${ }^{1}$, Pallavi S Joglekar ${ }^{2}$ and AmolS Patil ${ }^{3}$.

1. Intern, Bharati Vidyapeeth Deemed University Dental College \& Hospital, Pune, India.

2. Intern, Sinhgad Dental College\& Hospital, Pune, India.

3. Professor, Department of Orthodontics, Bharati Vidyapeeth Deemed University Dental College \& Hospital, Pune, India.

\section{Manuscript Info}

(.........................

Manuscript History

Received: 15 July 2016

Final Accepted: 19 August 2016

Published: 16 September 2016

Keywords:-

Musculoskeletal disorders, occupational

hazards, dental students, dental

education, occupational health

\begin{abstract}
Objective: To compare the prevalence of musculoskeletal disorder in the dental undergraduate, postgraduate students and non-dental students as well as to evaluate the correlation between different risk factors and prevalence of musculoskeletal disorders.

Material and methods: Total 480 questionnaires were answered; 320 questionnaires were answered by 160 postgraduates and 160 undergraduates from 6 different departments; orthodontics, prosthodontics, periodontics, pedodontics, oral surgery, and endodontics in two dental colleges in Pune, India. The control group consisted of 160 non-dental students from the similar age groups from the same Universities. The answers were statistically analyzed using SPSS through Chisquare test, Kruskal Wallis test and post hoc test. Logistic regression was analyzed to prove the correlation between the risk factors such as age, gender, departments, total years of dental practice, number of patient treated per working day, etc.

Results: Amongst the dental postgraduate groups, neck (52.5\%) was found to be most common musculoskeletal disorder followed by lower back $(43.33 \%)$, shoulder $(35.83 \%)$ and upper back (32.29\%). However, undergraduate students had more of back pain followed by neck and shoulder pain. Treatment resorted for the musculoskeletal pain was proportional to the prevalence of musculoskeletal disorders in each groups. Logistic regression analysis has shown that neck pain was associated with no break in between each patient session. Time spent in each patient appointments were strongly associated with upper and lower back pains.

Conclusion: There is high prevalence of musculoskeletal disorders associated with dental students even in their early dental college training years. Higher prevalence in undergraduates in most of musculoskeletal disorders except for neck suggests that young dentists in their college years resort to bending of their upper and lower back more often and they have improper posture of their upper extremities during the procedure. Since the musculoskeletal disorders are the most prevalent occupational hazards of dental professionals, appropriate postures from early training years are essential for the long career.
\end{abstract}

CopyRight, IJAR, 2016,. All rights reserved.
Corresponding Author:-Amol S Patil. MDS, PhD

Address:-Professor.Department of Orthodontics, Bharati Vidyapeeth Deemed University Dental College \& Hospital, Pune, India. 


\section{Introduction:}

Musculoskeletal pain has been found to be the most common occupational hazards of dental practitioners. In the survey study conducted on the self-reported occupational health of general dentist in New Zealand, almost $60 \%$ of general dentists reported that they have experienced pain or discomfort and close to $40 \%$ have required analgesics at least once in the previous 4 weeks. ${ }^{[1]}$ The similar studies conducted in Finland and Southern Thailand also have shown the prevalence of musculoskeletal disorders as high as $70 \%$ and $78 \%$ respectively. ${ }^{[2][3]}$

Such high prevalence of musculoskeletal disorders may be due to combination of awkward position caused by flexion of back, neck and less movements of head, back and upper arms required during the patient treatment for high precision. ${ }^{[4]}$ This static and awkward posture as well as repetitive movements, high pretension forces and long work duration has been identified as the biomechanical risk factor associated with the development of musculoskeletal disorders among dentists. ${ }^{[5]}$

Even though many researches are reported on the prevalence of musculoskeletal disorders in general dentists and ergonomics has been developed to prevent it by improving the posture of the dentist, there have been deficient number of studies that reports the prevalence of such disorders in dental students. Hence, the present study is designed to evaluate the prevalence of musculoskeletal disorders in dental undergraduate, postgraduate students and non-dental students as well as the differences between dental undergraduate and postgraduate groups to analyze the underlying risk factor of musculoskeletal disorders.

\section{Materials and Methods:}

The Standardized Nordic questionnaire was used to acquire the data regarding the experience of any kind of discomfort in different anatomical regions, duration of musculoskeletal pains, treatment sought, etc. ${ }^{[22]}$ The risk factors such as total years of dental practices, working hours per day, number of patient treated per day and duration of break between each patient session were also assessed in this questionnaire.

Total 320 questionnaires were collected from the volunteers in two dental colleges; 160 postgraduates, 160 undergraduates. For assessment of undergraduate group, interns were selected because they have more clinical exposure than any other years of undergraduate dental course in India. 160 questionnaires were completed by nondental students of similar age from same University campuses. The dental postgraduate volunteers were selected from 6 clinical specialties, viz. Orthodontics, Periodontics, Oral Surgery, Pedodontics, Endodontics and Prosthodontics. Interns have 1-month rotatory posting in concordance with dental curriculum of Indian Dental Council. The volunteers for dental undergraduate group were also from the same departments as it was mentioned for dental postgraduates.

After 1-month of data collection, data were entered in Excel and SPSS v20 software(IBM Corp. Released 2011. IBM SPSS Statistics for Windows, Version 20.0. Armonk, NY) was used to analyze the primary data, to perform the chi-square test, Kruskal Wallis test and post hoc Dunn-Bonferroni test.Also logistic regression was analyzed to check the correlation of different factors to the prevalence of musculoskeletal disorders in dental groups.

\section{Results:}

The sample size for different groups were 160 each; comprising of 320 dental group ( 160 dental postgraduates and 160 undergraduates) and 160 control group. Out of 480 questionnaires that were answered, $148(30.83 \%)$ were men and $332(69.16 \%)$ were women. Their mean age was 25.94 years $( \pm 2.18)$ for postgraduates, 22.91 years $( \pm 1.26)$ for undergraduates and 24.04 years $( \pm 1.70)$ for the non-dental students. 320 dental students were from different departments viz. orthodontics $(13.75 \%)$, periodontics $(16.25 \%)$, oral surgery $(20.35 \%)$, pedodontics $(12.81 \%)$, endodontics (21.56\%) and prosthodontics (15.31\%)

Table 1:-

\begin{tabular}{|l|l|l|l|l|l|l|l|l|l|}
\hline & \multicolumn{7}{|c|}{ Prevalence of Musculoskeletal Disorders } \\
\hline & Neck & Lower Back & Upper back & Shoulder & Wrist & Knee & Ankle & Elbow & Hips \\
\hline PG & $52.50 \%$ & $43.33 \%$ & $32.29 \%$ & $35.83 \%$ & $13.54 \%$ & $6.04 \%$ & $12.92 \%$ & $5.00 \%$ & $5.42 \%$ \\
\hline UG & $39.79 \%$ & $45.63 \%$ & $43.54 \%$ & $38.13 \%$ & $25.00 \%$ & $6.25 \%$ & $5.42 \%$ & $4.38 \%$ & $3.54 \%$ \\
\hline Control & $21.25 \%$ & $21.67 \%$ & $23.33 \%$ & $15.42 \%$ & $12.71 \%$ & $4.38 \%$ & $5.00 \%$ & $3.96 \%$ & $3.13 \%$ \\
\hline
\end{tabular}


Graph 1:-

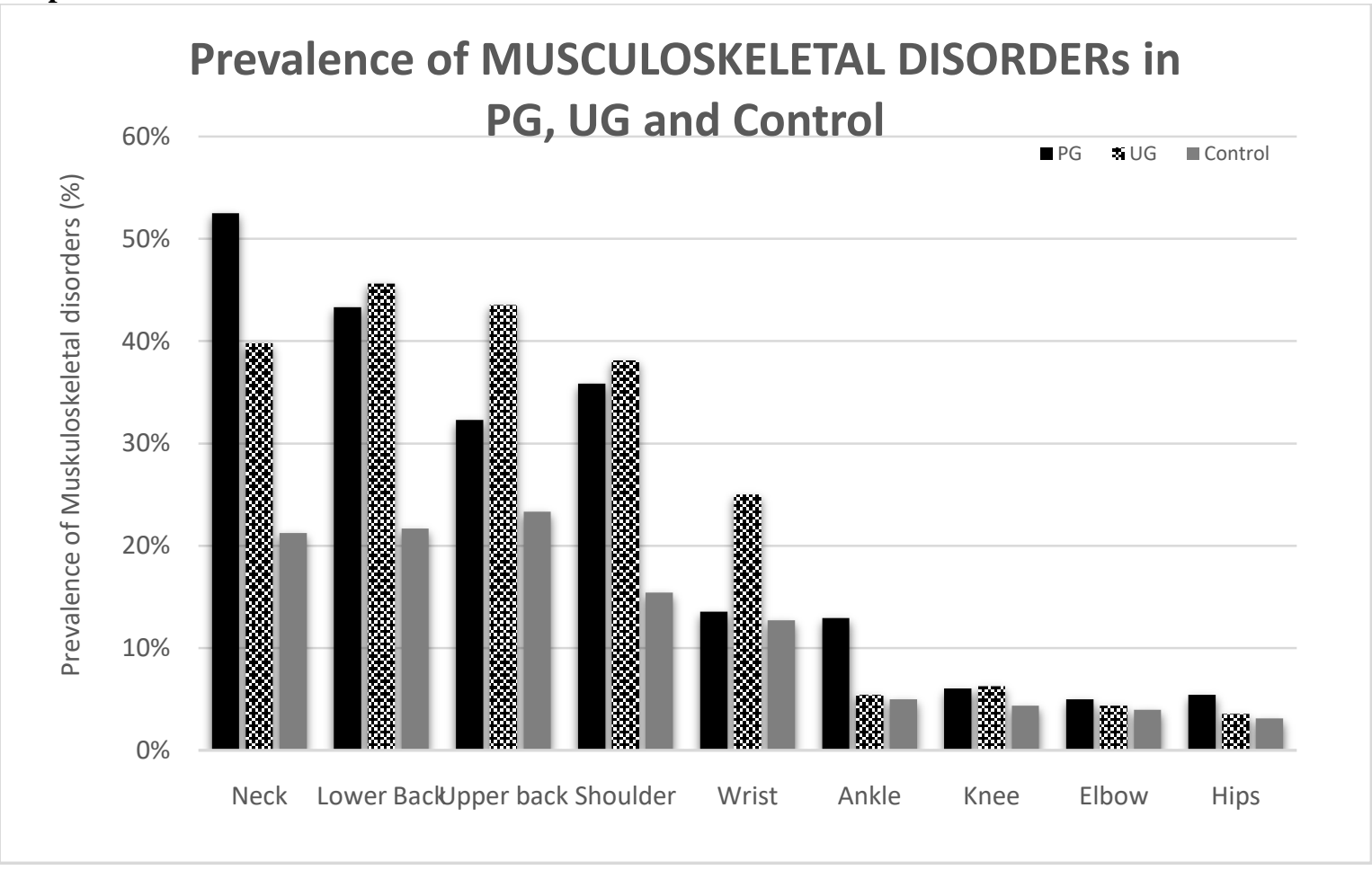

Table 2:- Chi-Square test.

\begin{tabular}{|c|c|c|c|l|c|c|c|c|c|}
\hline \multicolumn{10}{|c|}{ Test Statistics $^{\mathbf{a}, \mathbf{b}}$} \\
\hline & Neck & $\begin{array}{l}\text { Lower } \\
\text { Back }\end{array}$ & $\begin{array}{l}\text { Upper } \\
\text { Back }\end{array}$ & Shoulder & Wrist/Hands & Ankle & Knee & Hips/Thighs & Elbow \\
\hline Chi-Square & 32.521 & 17.206 & 11.620 & 18.137 & 10.141 & 27.210 & 1.248 & 0.513 & 0.377 \\
\hline df & 2 & 2 & 2 & 2 & 2 & 2 & 2 & 2 & 2 \\
\hline Asymp. Sig. & $\begin{array}{c}0.00 \\
0\end{array}$ & 0.000 & 0.003 & 0.000 & 0.006 & 0.000 & 0.536 & 0.774 & 0.828 \\
\hline
\end{tabular}

Prevalence of musculoskeletal disorders in different groups are shown in Table no. 1. Prevalence of musculoskeletal disorders was assessed through answer of questions such as 'Have you ever had any trouble?', 'Have you ever had such trouble in last 12 months', 'what is the total length of time you had the trouble in the last 12 months', 'what is the total length of time that trouble has prevented you from doing your normal work (at home or away from home) during the last 12 months?' and 'Have you had the pain in last 7 days?'; the answer 'no' was coded 1 and 'yes' was coded 2. Also for questions which asked the total length of such troubles the answer '0days' were coded 1 and other options which signified the occurrence were coded 2.

For postgraduates the most common musculoskeletal disorders were neck, lower back, shoulder, upper back, wrist and ankle in sequence. However, undergraduates had most common experience of pain in both upper and lower back followed by neck, shoulder and wrist. The results are plotted in clustered bar graph for better understanding (Graph no. 1). The responses were statistically analyzed using Kruskal Wallis test and all the musculoskeletal disorders were shown to be statistically significant for 3 groups except knee, hips and elbow (Table 2). The post hoc DunnBonferroni test was also conducted and was found that all the musculoskeletal disorders reject the null hypothesis except elbow, hips and knee.

The risk factors that was studied in present studies were total years of dental practice, working hours per day, working hours per weeks, whether or not taking a break in between the patient sessions, duration of break, number of patient treated per day and duration of each patient session. The results and percentage of responses are shown in 
Table 3:

\begin{tabular}{|c|c|c|c|c|c|c|c|}
\hline & \multicolumn{2}{|r|}{ PG } & \multicolumn{2}{|c|}{ UG } & \multicolumn{2}{|c|}{ Total } \\
\hline & & Count & Percentage & Count & Percentage & Count & Percentage \\
\hline \multirow{3}{*}{$\begin{array}{l}\text { Duration of each } \\
\text { patient session }\end{array}$} & $30 \mathrm{~min}$ & 52 & $16.25 \%$ & 49 & $15.31 \%$ & 101 & $31.56 \%$ \\
\hline & $30-60 \mathrm{~min}$ & 101 & $31.56 \%$ & 102 & $31.88 \%$ & 203 & $63.44 \%$ \\
\hline & more than 60 min & 7 & $2.19 \%$ & 9 & $2.81 \%$ & 16 & $5.00 \%$ \\
\hline \multirow[t]{2}{*}{ Break } & No & 48 & $15.00 \%$ & 29 & $9.06 \%$ & 77 & $24.06 \%$ \\
\hline & Yes & 112 & $35.00 \%$ & 131 & $40.94 \%$ & 243 & $75.94 \%$ \\
\hline \multirow{4}{*}{$\begin{array}{l}\text { Number } \begin{array}{r}\text { of } \\
\text { patient } \\
\text { per day }\end{array} \\
\text { treated }\end{array}$} & $1-5$ patients & 116 & $36.25 \%$ & 137 & $42.81 \%$ & 253 & $79.06 \%$ \\
\hline & 6-10 patients & 39 & $12.19 \%$ & 18 & $5.63 \%$ & 57 & $17.81 \%$ \\
\hline & 11-15 patients & 5 & $1.56 \%$ & 2 & $0.63 \%$ & 7 & $2.19 \%$ \\
\hline & $\begin{array}{l}\text { more than } 15 \\
\text { patients }\end{array}$ & 0 & $0.00 \%$ & 3 & $0.94 \%$ & 3 & $0.01 \%$ \\
\hline
\end{tabular}

Table 4-

\begin{tabular}{|l|l|l|l|l|l|l|}
\hline \multirow{2}{*}{} & \multicolumn{2}{|c|}{ PG } & \multicolumn{2}{|c|}{ UG } & \multicolumn{2}{|c|}{ Total } \\
\cline { 2 - 7 } & Mean & $\begin{array}{l}\text { Standard } \\
\text { Deviation }\end{array}$ & Mean & $\begin{array}{l}\text { Standard } \\
\text { Deviation }\end{array}$ & Mean & $\begin{array}{l}\text { Standard } \\
\text { Deviation }\end{array}$ \\
\hline Total years of dental practice & 4.78 & 2.36 & 1.43 & 0.50 & 3.10 & 2.39 \\
\hline Working hours per day & 9.46 & 7.28 & 6.74 & 1.56 & 8.10 & 5.43 \\
\hline Working hours per week & 41.95 & 13.05 & 37.77 & 10.80 & 39.86 & 12.14 \\
\hline Duration of break & 11.02 & 14.65 & 11.74 & 12.89 & 11.38 & 13.78 \\
\hline
\end{tabular}

Table no. 3 \& Table no. 4. Total years of dental practice was 4.78 years $( \pm 2.36)$ for postgraduate whereas it was 1.43 years $( \pm 0.50)$ for undergraduates. There was also the difference in working hours per day as well as working hours per week in postgraduate and undergraduate groups. The number of patients each group treated per day were generally more in postgraduates than undergraduates and none of the group treated more than 15 patients except the negligible results shown in undergraduate group. The postgraduates had more percentage of "no" to the question that asked whether or not they take the break and even if they had, it was of lesser duration than the undergraduates. Collectively, the intensity of the work was more intense in the dental postgraduates as one would expect in both the aspects of number of patients treated and duration of break

Logistic regression was analyzed using SPSS to determine the univariate and multivariate association between each musculoskeletal disorder and the above mentioned factors in dental undergraduate and postgraduate groups. (Table 5, $6 \& 7$ ) The pain during last 12 months were assessed through the answer options such as 0 days, 1-7 days, 8-30 days, more than 30 days. As the present study concentrates more on the prevalence of musculoskeletal disorders in the dental students, 0 days were coded 1 and the other options which signified the presence of musculoskeletal disorder in any intensity were coded 2 . The result has shown that the male dental students were more prone to have neck, shoulder, upper back and wrist musculoskeletal pain. Age of the student was found to be probably statistically significant in the prevalence of musculoskeletal disorder in neck, shoulder and wrist. Total years of dental practice were found to be associated with the incidence of upper back, shoulder and wrist pain. Working hours per days showed the association with only shoulder and wrist. However, working hours per weeks showed the significance in all musculoskeletal disorders except wrist.

Treating 6-10 patients per day was weakly associated with incidence the neck pain. For upper back pain however it showed a high odds ratio irrespective of number of patient treated. However, p-values were $0.16,0.22$ and 0.14 respectively and they were statistically non-significant. Shoulder and wrist also showed statistically significant results but their odds ratio was found to be negligible. Neck pain was related to different departments such as orthodontics, periodontics, pedodontics and endodontics. Lower back pain showed correlation with orthodontics and endodontic departments. Likewise, upper back pain had association with pedodontics. Wrist was found to be associated with orthodontics department. Even though Upper and lower back did not show any apparent relationship with the departments, they along with the shoulder showed statistically significant association with the time spent in each patient appointment with their odds ratio above 2 . Neck pain was found to be highly significant when no break was taken during the patient sessions. 
Table 5: Risk factors and neck, lower back pain in past 12 months (Univariate analysis)

\begin{tabular}{|l|l|l|l|l|}
\hline Risk Factors & Neck Pain OR & $95 \%$ CI & Lower Back Pain OR & $95 \%$ CI \\
\hline Gender (Male) & $0.77^{*}$ & $0.47-1.28$ & 1.09 & $0.64-1.85$ \\
\hline Age & $1.11^{*}$ & $0.97-1.26$ & 0.98 & $0.85-1.13$ \\
\hline Total years of Experience & 1.02 & $0.90-1.16$ & 1.04 & $0.91-1.19$ \\
\hline Working hours per day & 0.99 & $0.94-1.03$ & 1.00 & $0.96-1.05$ \\
\hline Working hours per week & 0.99 & $0.98-1.01$ & 1.00 & $0.98-1.02$ \\
\hline Duration of Break & $1.01^{*}$ & $0.99-1.02$ & $1.01^{*}$ & $0.99-1.03$ \\
\hline
\end{tabular}

*Probably statistically significant (p-value $<0.5) * *$ Statistically significant(p-value $<0.05)$

Table 6:Risk factors and upper back, shoulder and wrist pain in past 12 months (Univariate analysis)

\begin{tabular}{|l|l|l|l|l|l|l|}
\hline Risk Factors & Upper Back Pain OR & $95 \%$ CI & Shoulder OR & $95 \%$ CI & Wrist OR & $95 \%$ CI \\
\hline Gender (Male) & $0.75^{*}$ & $0.42-1.33$ & $1.40^{*}$ & $0.80-2.43$ & $1.43^{*}$ & $0.68-3.01$ \\
\hline Age & 1.00 & $0.87-1.16$ & $0.95^{*}$ & $0.81-1.10$ & $0.74^{* *}$ & $0.57-0.96$ \\
\hline Total years of Experience & $0.95^{*}$ & $0.82-1.09$ & $1.11^{*}$ & $0.96-1.27$ & $1.12^{*}$ & $0.91-1.38$ \\
\hline Working hours per day & 0.99 & $0.94-1.05$ & $0.93^{*}$ & $0.86-1.01$ & $0.89^{*}$ & $0.75-1.07$ \\
\hline Working hours per week & $0.98^{*}$ & $0.96-1.00$ & $0.99^{*}$ & $0.97-1.01$ & 0.99 & $0.96-1.03$ \\
\hline Duration of Break & $0.99^{*}$ & $0.97-1.01$ & 1.00 & $0.98-1.02$ & 0.99 & $0.96-1.02$ \\
\hline
\end{tabular}

*Probably statistically significant (p-value $<0.5) * *$ Statistically significant $(\mathrm{p}$-value $<0.05)$

Treatment sought in any form such as taking analgesics or appointment with doctors for different musculoskeletal disorders were tabulated in Table no. 8. Amongst the PGs, treatment sought for neck pain was the highest $(36.87 \%)$, followed by treatment for lower back pain(30\%). However, amongst the UGs $25 \%$ sought treatment for lower back pain and $20 \%$ for neck pain. In control group relatively lesser number of students have sought the treatment than the dental students.

\section{Discussion:}

Out of 480 dental and non-dental students almost 59\% have complained of musculoskeletal pain in atleast one area of the body. Such high prevalence is identical with other studies. ${ }^{[1][2][3]}$ However, the present study is of particular importance since this is one of few studies that has been designed to compare the dental undergraduates and postgraduates as well as dental group from non-dental group.

Prevalence of musculoskeletal disorders in the postgraduates followed the sequence of neck, lower back, shoulder, upper back, wrist, ankle, and knee, elbow hips being negligible. However, for the undergraduates except for neck it outpaced most of the symptoms, viz. lower back, upper back, shoulder, wrist. Lower back and upper back were found to be most commonly affected followed by neck shoulder and wrist which indicates inability of undergraduate students to maintain proper posture during the procedures which could be due to lesser experience and training. The control group exhibited relative regular distribution in the prevalence of all musculoskeletal disorders. Kruskal Wallis test have shown that the data in between all three groups were statistically significant except for knee, hips, and elbows. Post hoc Dunn-Bonferroni test has proved the same result. Even though the data on the dental students are lacking there have been many studies that have shown the prevalence of musculoskeletal disorders in different countries and the results are similar to the present study. ${ }^{[1][6][7][8][9][11][12]}$ Some studies have shown that the most common musculoskeletal disorders to be neck, lower back and shoulder ${ }^{[1][6][7][9]}$ whereas others have shown lower back as the most common followed by neck and shoulder, ${ }^{[8][11][12]}$ which is seen in undergraduate students of the present study. However some studies describe shoulder as the second common musculoskeletal disorders followed by back $^{[2][13][14]}$

In the present study, the males were more prone to have musculoskeletal disorders. This result is similar to the study undertaken by Ayers et al ${ }^{[1]}$. However, the other studies have shown that females have more musculoskeletal symptoms ${ }^{[15-21]}$ which they attributed to different constitution of women, higher susceptibility or lower resistance to constant strain, smaller muscule strength, etc ${ }^{[8]}$.

Age, total years of experience, working hours per day, departments and number of patients treated per working days were probably statistically significant $(\mathrm{P}<0.5)$ hence no concrete relationships could be dictated by the results $($ Table no. 5-7). However, when no break was taken neck pain had 1.79 odds ratio with CI 1.01-3.16 and it was found to be 

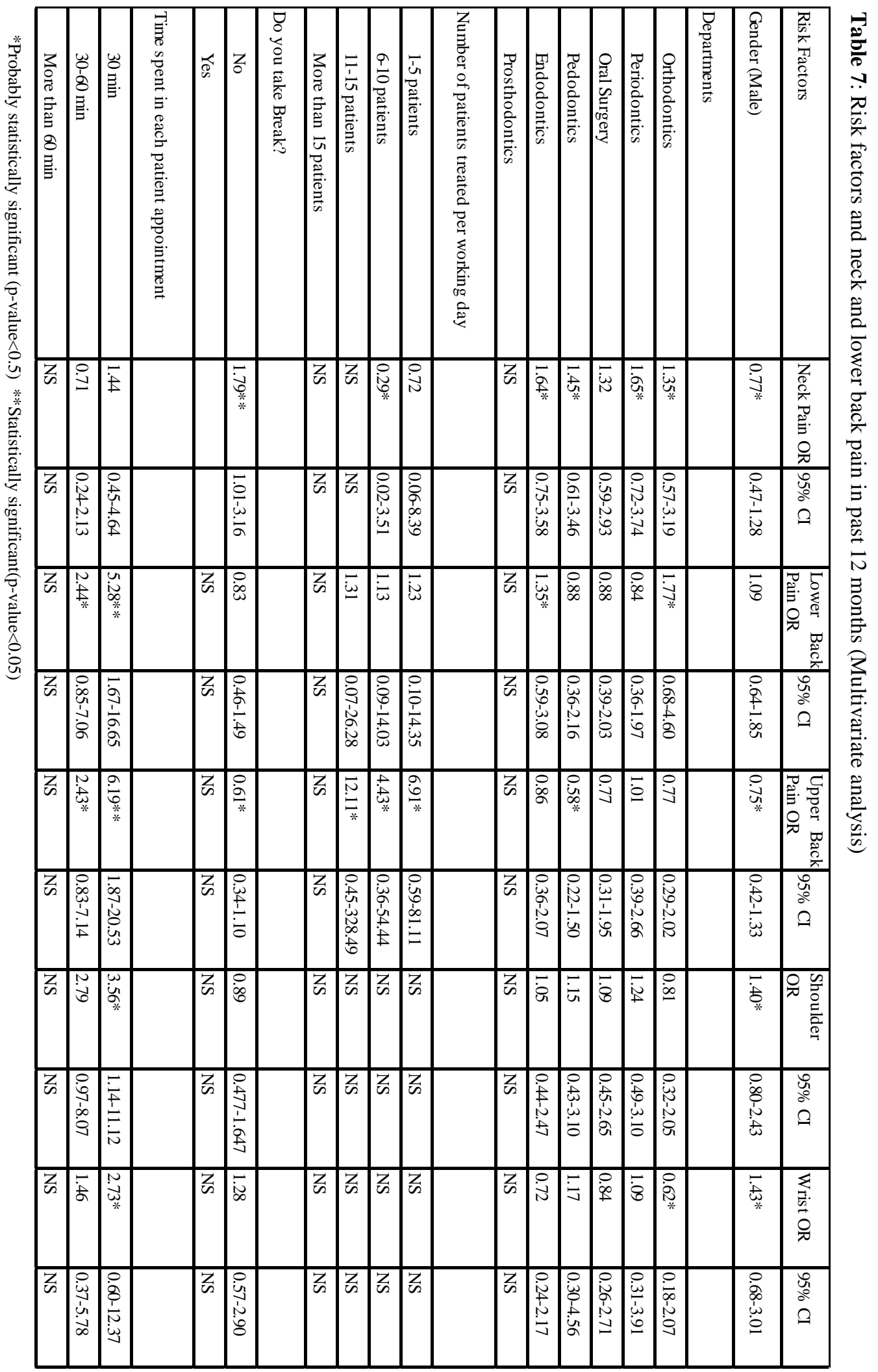
Table 8: Treatment sought (analgesics/treatment from doctor)

\begin{tabular}{|l|l|l|l|l|l|l|l|l|l|}
\hline & Neck & Shoulder & Upper Back & Elbow & Lower Back & Wrist & Hip & Knee & Ankle \\
\hline \multirow{3}{*}{ PG } & 59 & 22 & 30 & 1 & 48 & 10 & 3 & 4 & 10 \\
\cline { 2 - 11 } & $36.87 \%$ & $13.75 \%$ & $18.75 \%$ & $0.625 \%$ & $30.00 \%$ & $6.25 \%$ & $1.875 \%$ & $2.5 \%$ & $6.25 \%$ \\
\hline \multirow{2}{*}{ UG } & 32 & 25 & 24 & 3 & 40 & 11 & 0 & 4 & 4 \\
\cline { 2 - 11 } & $20 \%$ & $15.63 \%$ & $15.10 \%$ & $1.88 \%$ & $25 \%$ & $6.88 \%$ & $0.00 \%$ & $2.5 \%$ & $2.5 \%$ \\
\hline \multirow{2}{*}{ Control } & 20 & 16 & 18 & 5 & 13 & 9 & 0 & 7 & 10 \\
\cline { 2 - 11 } & $12.5 \%$ & $10 \%$ & $11.25 \%$ & $1.04 \%$ & $3.13 \%$ & $31.64 \%$ & $0.00 \%$ & $4.37 \%$ & $6.25 \%$ \\
\hline
\end{tabular}

statistically significant. Also when time spent in each patient appointments were 30 minutes, the OR for upper and lower back pain were 6.19 and 5.28 respectively and they were found to be statistically significant. These results are consistent with the Greek study which have studied the relationship of different risk factors such as age, working long hours, etc. to the prevalence of musculoskeletal disorders in the dentists. ${ }^{[22]}$ Alexopoulos et al also have found the age and the longer working hours are not statistically significant. ${ }^{[22]} \mathrm{Yi}$ et al. have studied the different risk factors for postgraduates in different specialty and stated that years of clinical work was correlated with the presence of musculoskeletal disorders in orthodontics, prosthodontics and periodontics. ${ }^{[14]}$ Since the present study was designed to evaluate the risk factors in both the undergraduate and postgraduate group as dental student group as a whole, it is assumed that our results did not show the similar result.

Lastly the Table no. 8 indicates that the number of responses who have sought any form of treatment corresponds to the most common musculoskeletal disorders. The treatment sought for neck pain was highest $(36.87 \%)$ in dental postgraduate groups followed by lower back $(30 \%)$, upper back (18.75\%), shoulder (13.75\%),etc. However, in dental undergraduate groups lower back (25\%) was followed by neck (20\%), shoulder (15.63\%), upper back(15.10\%), etc. Control group had relatively lower percentage of treatments that were required for the musculoskeletal pains.

\section{Conclusion:}

There is high prevalence of musculoskeletal disorders associated with dental students even in their early dental college training years. Higher prevalence in undergraduates in most of musculoskeletal disorders except for neck suggests that young dentists in their college years resort to bending of their upper and lower back more often and they have improper posture of their upper extremities during the procedure. Since the musculoskeletal disorders are the most prevalent occupational hazards of dental professionals, appropriate postures from early training years are essential for the long career.

\section{Acknowledgement:-}

We acknowledge all the volunteers who have filled the questionnaires for successful collection of the data.

Contribution of the authors.

\begin{tabular}{|l|c|c|c|c|}
\hline & JH Kim & N Raval & P Joglekar & A Patil \\
\hline Designing \&concept development & $\sqrt{ }$ & $\sqrt{ }$ & \\
\hline Data collection and entry & $\sqrt{ }$ & $\sqrt{ }$ & \\
\hline Manuscript writing & $\sqrt{ }$ & $\sqrt{ }$ & $\sqrt{ }$ \\
\hline Manuscript Revision \& editing & $\sqrt{ }$ & $\sqrt{ }$ & & $\sqrt{ }$ \\
\hline Statistical Analysis & $\sqrt{ }$ & & & \\
\hline
\end{tabular}




\section{References:}

1. AyersK. M. S, ThomsonW. M, NewtonJ. T, MorgaineK. C., RichA. M. (2009): Self-reported occupational health of general dental practitioners; occupational medicine; Occupational Medicine 59:142-148

2. KerosuoE, KerosuoH, KanervaL (2000): Self-reported health complaints among general dental practitioners, orthodontist, and office employees; Acta Odontol Scand 58:207-212.

3. ChowanadisaiS, KukiattrakoonB, YapongB, KedjaruneU(2000): Occupational health problems of dentists in southern Thailand; International Dental Journal vol. 50/No.1

4. JonkerD, RolanderB, BaloghI, SandsjoL, EkbergK, WinkelJ(2011): Mechanical exposure among general practice dentists in Sweden and possible implications of rationalization; Ergonomics, 54:10, 953-960

5. Sanders M, Turcotte C (1997): Ergonomic strategies for dental professionals; Work 8:55-72

6. LeggatPA, SmithDR(2006): Musculoskeletal disorders self-reported by dentists in Queensland, Australia; Australian Dental journal51:4

7. BothaPJ, ChikteU, BarrieR, EsterhuizenTM(2014): Self-reported musculoskeletal pain among dentists in South Africa: A 12-month prevalence study; SADJVol 69;5: p208-p213

8. HodacovaL, SustovaZ, CermakovaE, KapitanM, SmejkalovaJ(2015): Self-reported risk factors related to the most frequent musculoskeletal complaints among Czech dentists; Industrial Health 53, 48-55

9. SustovaZ, HodacovaL, KapitanM(2013): The prevalence of musculoskeletal disorders among dentists in the Czech Republic; Acta Medica, 56(4):150-156

10. KazangiogluH O, BereketM C, EzirganliS, OzsevikS, SenerI(2013): Musculoskeletal complaints among oral and maxillofacial surgeons and dentists: A questionnaire study; Acta Odontol Scand, 71: 469-474

11. AljanakhM, ShaikhS, SiddiquiA A, Al-MansourM, HassanS S(2015): Prevalence of musculoskeletal disorders among dentists in the Ha'il Region of Saudi Arabia; Ann Saudi Med 35(6): 451-461

12. Khalid Al-Ali, Raghad Hashim (2012): Occupational health problems of dentists in the United Arab Emirates; Int Dent J62:52-56

13. RafeemaneshE, JafariZ, KashaniF O, RahimpourF(2013): A study on job postures and musculoskeletal illnesses in dentists; IJOMEH 26(4)

14. YiJ, HuX, YanB, ZhengW, LiY, ZhaoZ (2013): High and specialty-related musculoskeletal disorders afflict dental professionals even since early training years; J Appl Oral Sci 21 (4): 376-82

15. Shrestha BP, Singh GK, Niraula SR (2008): Work related complaints among dentists. JNMA J Nepal Med Assoc 47, 77-81.

16. Lindfors P, von Thiele U, Lundberg U (2006): Work characteristics and upper extremity disorders in female dental health workers. J Occup Health 48, 192-7.

17. Puriene A, Aleksejuniene J, Petrauskiene J, Balciuniene I, Janulyte V (2008): Self-reported occupational health issues among Lithuani

18. Akesson I, Johnsson B, Rylander L, Moritz U, Skerfving S (1999): Musculoskeletal disorders among female dental personnel-clinical examination and a 5-year follow-up study of symptoms. Int Arch Occup Environ Health 72, 395-403. Harutunian K, Gargallo-Albiol J, Figueiredo R, Gay- Escoda C (2011): Ergonomics and musculoskeletal pain among postgraduate students and faculty members of the School of Dentistry of the University of Barcelona (Spain). A cross-sectional study. Med Oral Patol Oral Cir Bucal 16, e425-9.

19. Sartorio F, Vercelli S, Ferriero G, D’Angelo F, Migliario M, Franchignoni M (2005): Work-related musculoskeletal diseases in dental professionals. 1. Prevalence and risk factors. G Ital Med Lav Ergon 27, 1659 (in Italian).

20. Valachi B (2008): Musculoskeletal health of the woman dentist: distinctive interventions for a growing population. J Calif Dent Assoc 36, 127-32.

21. AlexopoulosE C, StathiI, CharizaniF(2004): Prevalence of musculoskeletal disorders in dentists; BMC Musculoskeletal Disorders 5:16; pg 4

22. Kuorinka I, Jonsson B, Kilbom A, Vinterberg H, Biering-Sørensen F, Andersson G (1987): Standardised Nordic questionnaires for the analysis of musculoskeletal symptoms. Appl Ergon.18:233-7 